\title{
Fitting undeformed superquadrics to range data: improving model recovery and classification
}

\author{
Erik R. van Dop* \\ Paul P. L. Regtien* \\ Department of Electrical Engineering, University of Twente, The Netherlands
}

\begin{abstract}
Undeformed superquadrics are volumetric modeling primitives with an extensive shape vocabulary that are described by only 5 parameters. Fitting these models viewpoint invariantly to range data enables classification based on the superquadric parameters. However, current recovery routines show several limitations, especially when the algorithms are applied to range images instead of true $3 D$ images. In this paper problems with the common superquadric recovery procedure are identified and solutions are presented.
\end{abstract}

\section{Introduction}

Superquadric models have received substantial interest from several researchers [13] as a volumetric primitive for range image segmentation and object recognition. One reason is the viewpoint invariant recovery of the models and another is the extensive shape vocabulary of superquadrics, including cylinders, spheres, blocks and shapes in between (figure 1 [8]). By applying global deformations like tapering and bending to the undeformed model the representational power can increase even further [1, 14]. Despite their favorable properties, superquadric model fitting based on Solina's original algorithm [14] has not been implemented very successfully because it shows some severe limitations. Some of these arise only when range images $(2.5 D$ images) are considered (as opposed to true $3 D$ images), but other defects are encountered for both range and $3 D$ images. In this paper Solina's recovery procedure is analyzed for the case of undeformed superquadrics, together with alternatives offered by other researchers. Adaptations to the algorithm are made in three ways: The distance measure during iterative fitting is altered (section 3), a background constraint is added for superquadric recovery from range images (section 4) and the routine is made robust against outliers in

\footnotetext{
* Measurement Science and Instrumentation Group, P.O. Box 217, 7500 AE Enschede, The Netherlands, E-mail: erik@mi.el.utwente.nl
}

range data (section 5). Furthermore, an unbiased maximum likelihood classification method is proposed (section 6). Results on real range images illustrate the progress made in superquadric recovery (section 7) and the possibilities of recognizing objects within the shape vocabulary of undeformed superquadrics. This enables a more successful introduction of these primitives in computer vision as is argued in some concluding remarks.

\section{Superquadric recovery}

A superquadric surface is defined by the equation:

$$
1=\left(\left(\frac{x}{a_{1}}\right)^{\frac{2}{\epsilon_{2}}}+\left(\frac{y}{a_{2}}\right)^{\frac{2}{\epsilon_{2}}}\right)^{\frac{\epsilon_{2}}{\epsilon_{1}}}+\left(\frac{z}{a_{3}}\right)^{\frac{2}{\epsilon_{1}}}
$$

Parameters $a 1, a 2$ and $a 3$ define the size and $\epsilon_{1}$ and $\epsilon_{2}$ define the shape of the superquadric.

For objects separated from the background (by

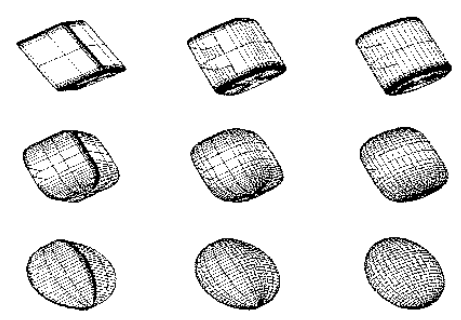

Figure 1: Superquadric models. From left to right $\epsilon_{2}=$ $0.1,0.5,1.0$, from top to bottom $\epsilon_{1}=0.1,0.5,1.0$.

thresholding), a robust algorithm for the superquadric recovery is known [4, 14]. In this algorithm the error of fit $(E O F)$ for the model is minimized in a nonlinear least-squares way (Levenberg-Marquardt). As an initial condition the center of gravity of the range data and the direction of the principal axes are chosen to define position and orientation of the initial coordinate system, with the Z-axis along the principal axis with least moment of inertia. Extremities along the estimated axes will be the starting point for the size estimates, while the shape estimates are initially set 
to 1 (an ellipsoid). Noise is added during minimization to prevent local minima to occur and $\epsilon_{1}$ and $\epsilon_{2}$ are restricted between 0.1 and 1 to avoid ambiguities in the recovered superquadrics.

\section{Distance measures}

Ideally, the distance metric of any model fitting process should be tailored to reflect the noise properties of the sensor in such a way that the fitting procedure would yield a maximum likelihood estimate [15]. For a range sensor corrupted with Gaussian noise, this implies a metric that uses the vertical distance $z$ of the data point to the surface. In the case of superquadric recovery there is no analytic solution of this distance as a function of the superquadric parameters and the noise model is usually not Gaussian. For this reason one has to settle for a sub-optimal distance metric. Solina [14] proposed a measure based on the so-called inside-outside function, but experiments by Gross [3] (on undeformed superquadrics) pointed out that a measure according to the radial distance from a data-point to the superquadric surface has less bias. These results can be validated theoretically by considering the parametric equation of a superquadric surface

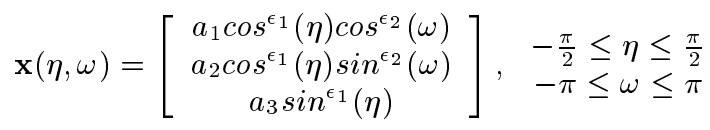

and its implicit form (or inside-outside function):

$$
f(\mathbf{x}, \mathbf{a})=\left(\left(\frac{x}{a_{1}}\right)^{\frac{2}{\epsilon_{2}}}+\left(\frac{y}{a_{2}}\right)^{\frac{2}{\epsilon_{2}}}\right)^{\frac{\epsilon_{2}}{\epsilon_{1}}}+\left(\frac{z}{a_{3}}\right)^{\frac{2}{\epsilon_{1}}}=1
$$

The radial distance $\delta$ (figure 2, $[15,5]$ ) of a datapoint to the superquadric surface can be expressed as

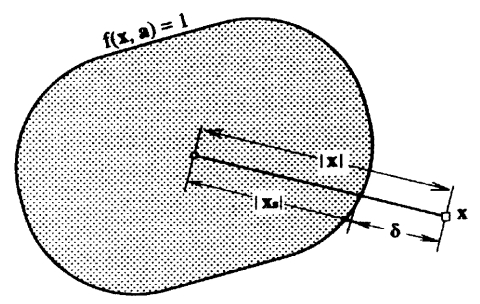

Figure 2: Radial distance measure $\delta$

the factor by which the superquadric has to be scaled to pass through the data-point.

$$
\mathbf{x}^{\prime}(\eta, \omega)=\left(1+\frac{\delta}{\left|\mathbf{x}_{\mathbf{s}}\right|}\right) \mathbf{x}(\eta, \omega)
$$

The relationship between $f(\mathbf{x}, \mathbf{a})$ and $\delta$ can then be written as:

$$
1+\frac{\delta}{\left|\mathbf{x}_{\mathbf{s}}\right|}=f(\mathbf{x}, \mathbf{a})^{\epsilon_{1} / 2}
$$

Solina's distance measure $D$ [14], incorporating the inside-outside function to the power $\epsilon_{1}$ and multiplied by a minimal volume criterion $V^{1 / 2}=\left(a_{1} a_{2} a_{3}\right)^{1 / 2}$ can now be expressed as a function of $\delta$ :

$$
D=V^{1 / 2}\left(f^{\epsilon_{1}}(\mathbf{x}, \mathbf{a})-1\right)=V^{1 / 2}\left(2 \frac{\delta}{\left|\mathbf{x}_{\mathbf{s}}\right|}+\frac{\delta^{2}}{\left|\mathbf{x}_{\mathbf{s}}\right|^{2}}\right)
$$

This equation states that data-points inside the superquadric contribute less to the distance measure than points outside the surface with the same $\delta$ and $\left|\mathbf{x}_{\mathbf{s}}\right|$, because the quadratic term partially compensates the distance measure of inside points while it amplifies that of for outside points. Furthermore, the main component in the error metric, $\frac{\delta}{\left|\mathbf{x}_{\mathbf{s}}\right|}$, decreases as $\left|\mathbf{x}_{\mathbf{s}}\right|$ increases, causing a bias towards superquadrics with large volumes. The volume criterion added by Solina compensates this bias, but as Gross [3] showed empirically this is only partially successful.

\section{Background constraint}

A main problem of superquadric recovery from single view range images is the size estimation in the viewing direction. This can be easily imagined: an ellipsoid with a main axis in the viewing direction has a range image that hardly depends on the elongation of the ellipsoid. The range image of a block showing only one face is even degenerate, i.e. a block of arbitrary size matches the range data.

Gupta [6] approaches this problem by extrapolating surfaces adjacent to the superquadric. When these surfaces are assumed to support the modeled object, data-points can be added to the segmentation routine and constrain the object size in the viewing direction (figure 3b). Unfortunately, the extrapolated points are usually not supporting points. To enhance the supporting plane estimation, Gupta defines a plane through the major axis of inertia of the superquadric and one of the other major axes, such that the angle between the normal of the plane and the direction of gravity is minimal. This plane is then shifted in the direction of gravity until it intersects with one of the earlier defined extrapolated points. Subsequently a set of data-points is generated for the supposed supporting plane (figure 3c). This approach has a fundamental discrepancy, because it falsely assumes that the points on the estimated supporting plane are true data-points.

In order to implement a supporting plane effectively, it should be treated as a constraint to the model-fitting process and not as a source of extra data-points. Since a supporting plane constraint has a highly nonlinear relationship with the fitting parameters it will be hard if not impossible to derive it analytically, thus disabling a parameter constraint 

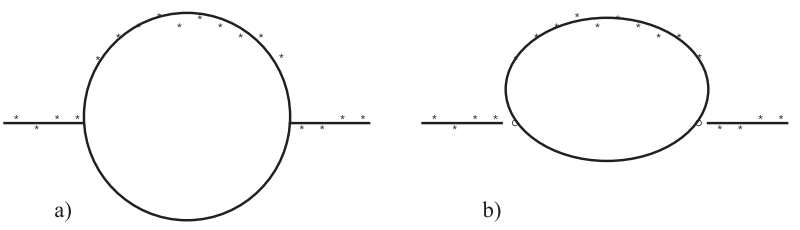

b)

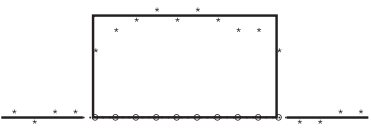

c)

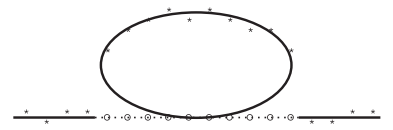

d)
Figure 3: Superquadric estimation: a) without background constraint; b) with added data-points (०) on extrapolated neighbouring plane; c) with added datapoints along the estimated support; d) with constraining points along the estimated support.

in the Levenberg-Marquardt minimalization process. The difficulty can be overcome by following Gupta's method of supporting plane estimation and treating the assumed supporting points as constraining points instead of data-points. Including only those estimated points in the model recovery that are inside the superquadric at the previous iteration, causes the superquadric to shrink such that the extrapolated points are pushed outside the superquadric. The weight attached to a constraining point is increased every iteration by a so-called penalty function [12] to ensure that the final recovery hardly includes such points, i.e. doesn't intersect the estimated supporting plane (figure $3 \mathrm{~d}$ ).

\section{Robust superquadric recovery}

Robustness is a factor frequently overlooked in computer vision algorithms, and the common superquadric recovery is not an exception. One single outlier in the data can cause an arbitrary change in the recovery, i.e. the procedure has a breakdown point (the smallest fraction of outliers in the dataset that can cause an arbitrarily large error) of zero. To tackle this problem, $\mathrm{Hu}$ [8] proposes an adaptive weighted partial data minimization algorithm which randomly selects a set of data-points with a small residual. This set of data-points is then used to estimate the updated superquadric. The idea behind this and most other well known robust minimization techniques like least median of squares (LMS) [11] is that the probability of selecting a data-set without outliers increases every iteration. When the data-points are distributed nicely around the complete superquadric this can be successful, but without this prerequisite it will fail. Consider for example the superquadric recovery from a single- view range image. Partial data selection introduces the possibility of ignoring points at the borders of the range image. A subsequent fitting procedure will result in a size underestimation of the superquadric that can increase every iteration.

As an alternative the least trimmed squares (LTS) estimator can be applied. It sorts the residuals and performs an iteration based on $(100-x) \%$ of the data with the smallest residuals, with $x$ as the maximal percentage of outliers in the dataset. This approach ensures that the fitting procedure uses a large fraction of the data when $x$ is small and stays the same throughout the whole procedure. The shrinking effect of the superquadric will therefore be limited. One important concern using LTS robust regression is its effect on the convergence of an algorithm Since only data-points that 'confirm' a previous fit are used to compute the next fit, it is more likely to end up in a local minimum.

To prevent this, the robust approach should start with a good initial estimate. Since the current algorithm uses data extremities for the initial size estimates this condition is not fulfilled. In order to obtain a better starting point for the superquadric recovery, a second LTS approach is proposed for the selection of datapoints used during initial estimation. Since outliers below the background level can be ignored right away and since outliers between the background level and the true surface are not very dangerous, only 'positive' outliers need to be revealed. This is easily done by selecting the $(100-y) \%$ data-points closest to the background.

Instead of trimming a fraction of the data, one can cancel out data-points above a threshold distance from the superquadric surface. This has the advantage that the computationally more intensive residual sorting is no longer necessary, but the threshold should be tuned very carefully in order to eliminate all the outliers but simultaneously prevent the shrinking effect.

Another class of robust estimators are the M- or bounded-influence estimators, but the breakdown point of these estimators deteriorates quickly as the number of estimated parameters increases [11]. In our case, where 11 parameters are estimated at a time this approach seems not very effective. The LTS approach is therefore implemented to equip the superquadric recovery with robustness.

\section{Classifying superquadrics}

Several attempts have been made towards superquadric classification based on its three size and two shape parameters. Some researchers use a decision function related to the (weighted) minimal Euclidean distance in parameter space between superquadric 
primitives and prototype models [7, 10]. Although this seems attractive from a cognition point of view, they result in biased classifiers because the conditional probability densities of the recovered parameters are neither independent nor Gaussian. Pentland [9] suggests a physics based measure indicating the amount of energy necessary to mold a superquadric such that it fits the prototype of an object. However, the relationship between elasticity coefficient of an object (representing the molding effort) and uncertainties in its superquadric representation is unclear, so classifier optimalization using this technique remains an open question.

Another decision function $\hat{\omega}(\vec{x})$, implemented by Whaite [15], uses a maximum likelihood criterion:

$$
\hat{\omega}(\vec{x})=\operatorname{argmax}\left\{p_{\vec{x} \mid \omega}(\vec{x} \mid \omega)\right\}
$$

where $p_{\vec{x} \mid \omega}(\vec{x} \mid \omega)$ is the conditional probability of a parameterization given a hypothesis and the conditional probabilities are assumed to have a multivariate normal distribution. This approach holds for small parameterization errors and a Gaussian error metric of the minimization process, but again both conditions are generally not met.

In reality the functional form of the probability density function is unknown, so it should be estimated to enable maximum likelihood classification [2]. A reliable estimation in a five dimensional feature space can only be acquired when an enormous training set is considered. For this reason the feature vector is mapped to a single parameter, by noting the relation between the conditional probability density of a parameter vector and the error surface of the noise-free object model. This can be understood by realizing that noise in the data perturbs the height of the (noise-free) error surface by some unpredictable, but bounded, amount. As a result the parameterization with minimum error found by the fitting procedure may shift. This shift is limited in directions where the error surface is steep but can be substantial when the error minimum lies in a shallow trough. By computing the error surface of a noise-free object model, an area in parameter space around the global minimum can be determined where the error surface is shallow. This is the area of parameterizations that can be found found when a superquadric is fitted to noisy data. Partitioning the five dimensional measurement space into bins, followed by off-line computation of an error surface for all noise free object models will thus be sufficient to determine a look-up-table of parameter vectors and their associated noise-free model error. This process is actually some sort of (non-linear) feature extraction method. Since the possible parameterizations of an object occupy only a small volume of the five dimensional space, the look-up-table remains limited in size when the bin size is not too small. A hash function can be used for indexing and quick retrieval of noise-free errors of fit associated with a recovered superquadric.

The estimation of the (one dimensional) conditional probability density function of the noise-free error of fit can be performed with a limited number of samples in the training set, but has to be done for every model separately because it is dependent on the number of data-points in the model. However, dividing the error of fit by the number of data-points results in the mean squared error of fit which is assumed to be model independent because all objects in the scene are subject to the same image noise. The validity of this assumption is shown in figure 4, where the conditional probability density function is estimated from training sets containing only one object class and for a training set containing several objects from every class. The distributions almost overlap, which implies model independency of the mean squared error.

A set of 100 real range images (of various models) was used to estimate the conditional probability density, using a nearest neighbour approach:

$$
\hat{p}_{\vec{x} \mid \omega}\left(\vec{x} \mid \omega_{k}\right)=\frac{\kappa}{N_{k} \cdot V(\vec{x})}
$$

where the number of samples in a neighbour region $(\kappa)$ is set to $3 \cdot \sqrt{N_{k}}, N_{k}$ is the number of samples in the training set (in our case 100) and $V(\vec{x})$ is the interval around $\vec{x}$ that just contains $\kappa$ samples.

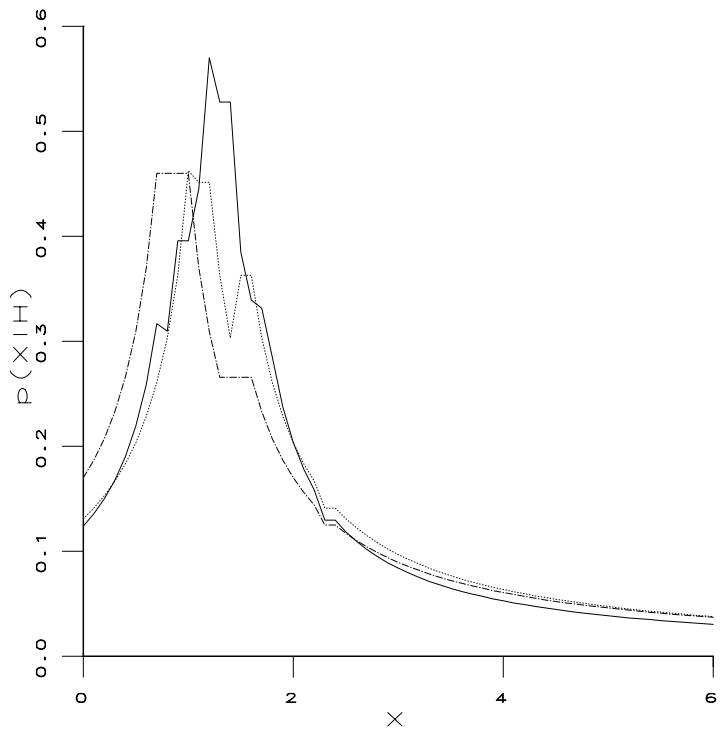

Figure 4: Estimated conditional probability density functions of the mean squared error of fit $(X)$ for $a$ training set of objects class $A$ (dotted) class $B$ (dashdotted) and a set containing all object classes (solid). 


\section{Experimental results}

The different versions of the superquadric recovery procedure, as presented in the previous sections, are analyzed by considering several realizations of range data of objects on printed circuit boards (figure 5).

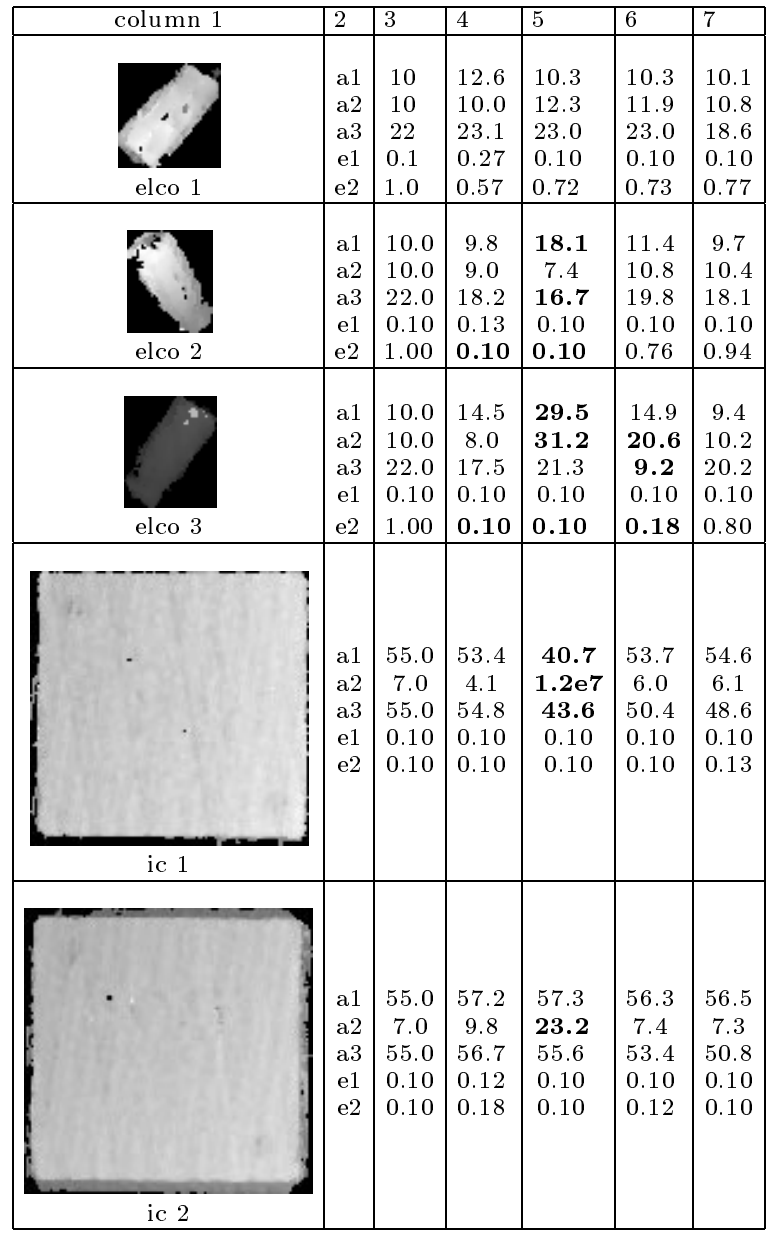

Figure 5: Superquadric recovery of several range images. Column 3 to \%: true parameters, results with the original (Solina's) algorithm, results after applying (Whaite's) radial distance measure, results after applying the radial distance measure and a background constraint, results after applying the radial distance measure, a background constraint and robustness.

It indicates that Solina's original procedure tends to recover 'squarish' superquadrics (small $e 1$ and $e 2$ ) and that it cannot deal with outliers (elco 3). Although Whaite's procedure (Solina's algorithm with a radial distance measure) succeeds in recovering better shape parameters it also fails when outliers are present and size estimation in the viewing direction is poor (already noted by Gross [3]). To resolve the latter problem, a background constraint was added (section 4). This results in a dramatic improvement of the superquadric recovery, but robustness (section 5 ) has to be added as well to allow outliers in the data. Other convincing indications of the improvements of the superquadric recovery are shown by the trace of the parameters over the course of 50 iterations (figure 6 ). The jaggedness of Solina's compared to Whaite's procedure shows the advantages of the radial distance measure, but without the background constraint and robustness the algorithm has a bad convergence. The improvement of the initial estimation with the LTS robustness approach becomes apparent as well.

Classification results as presented in a diffusion matrix (table 1) show that 85\% (123 from 144) of the objects in the database (electronic components like electrolytic capacitors, integrated circuits, crystals) are classified correctly. Since objects on a printed circuit board can have almost similar shapes and sizes (for example ic's with 10,12 or 14 leads) some classification errors still occur. The smallest objects are described by few hundred data-points.

\begin{tabular}{|c||c|c|c|c|c|c|c|c|c|c|c|c|c|}
\hline & 1 & 2 & 3 & 4 & 5 & 6 & 7 & 8 & 9 & 10 & 11 & 12 & $\mathrm{x}$ \\
\hline \hline 1 & 1 & & & & & & & & & & & & \\
\hline 2 & & 5 & & & & & & & & 2 & & & 1 \\
\hline 3 & & & 2 & & & & & & & & & & 1 \\
\hline 4 & & & & 3 & & & & & & & & & \\
\hline 5 & & & & & 1 & & & & & & & & \\
\hline 6 & & & & & & 2 & & & & & & & \\
\hline 7 & & & & & & & 7 & & & & & & 1 \\
\hline 8 & & & & & & & & 5 & & & & & \\
\hline 9 & & & & & & & & & 2 & & & & 1 \\
\hline 10 & & & & & & & & & & 23 & & & 8 \\
\hline 11 & & & & & & & & & & & 10 & & \\
\hline 12 & & & & & & & & & & & & 2 & \\
\hline $\mathrm{x}$ & 5 & & & 2 & & & & & & & & & 60 \\
\hline
\end{tabular}

Table1: Recognition results displayed as diffusion matrix (database objects 1 to 12 and unknown $x$ )

\section{Discussion}

Object recognition by superquadric representations has to be tailored to the scene and imaging system under consideration. This seems obvious, but careless implementation of the early algorithm has hampered application of superquadrics in vision systems. The lack of robustness and absence of an unbiased distance measure for classification have caused poor recognition results. Furthermore, the ignorance of constraints (for example defining the background plane) and the use of a suboptimal distance measure during model fitting limited the accuracy of the recovery. This paper provided solutions to these limitations for object recognition from range images in a scene with objects that can be modeled by undeformed superquadrics. Results on real data show recognition rates of $85 \%$ for objects closely resembling each other that are described by a few hundred data-points. However, a systematic 
analysis of the ability of superquadric classification should include extensive tests to relate image noise and superquadric parameter variations. Still, current results might restore some of the optimism about superquadric representations as a tool in computer vision, especially when the improvements are translated to the more general recovery and classification of globally deformed superquadrics.

\section{References}

[1] A. Barr, 'Global and local deformations of solid primitives', Computer Graphics, Vol. 18, no. 3, 21-30, 1984.

[2] E.R. van Dop, P.P.L. Regtien, 'Object recognition from range images using superquadric representations', IAPR workshop on machine vision appl., 267-271, 1996.

[3] A. Gross, T. Boult, 'Error of fit measures for recovering parametric solids', 2nd int. conf. on CV, 690-694,1988

[4] A. Gupta, R. Bajcsy, 'Volumetric segmentation of range images of 3D objects using superquadric models', CVGIP: Image understanding, vol. 58, No. 3, 302-326, 1993.

[5] A.Gupta,L.Bogoni,R.Bajcsy,'Quantitative and Qualitative measures for the evaluation of the superquadric models',IEEE $W$. on interpr. of $3 D$ scenes,162-169,1989

[6] A. Gupta, G. Funka-Lea, K. Wohn - 'Segmentation modeling and classification of the compact objects in a pile', Intelligent robots and computer vision VIII , 98-107,1989

[7] T. Horikoshi, H. Kasahara, '3-D shape indexing language', IEEE conf. on robotics and automation, 1662-1667,1992

[8] Y. Hu, W. Wee, 'Robust 3D part extraction from range images with deformable superquadric models',SPIE signal proc., sensor fus. and target recogn. IV,524-535,1995

[9] A. Pentland, S. Sclaroff, 'Modal representations', IEEE W on 3D-object representations in $C V, 249-262,1995$

[10] N.S. Raja, A.K. Jain, 'Recognizing geons from superquadrics fitted to range data', Image and vision computing, vol. 10, no. 3, 179-190, 1992.

[11] P. Rousseeuw, A. Leroy - Robust regression and outlier detection, Wiley, New York, 1987.

[12] L.E. Scales, Introduction to non-linear minimization, Macmillan, London, 1985.

[13] F. Solina,'Volumetric models in computer vision - an overview', J. of comp. and inf. tech.,no.3, 155-166,1994

[14] F. Solina, R. Bajcsy, 'Recovery of parametric models from range images: the case for superquadrics with global deformations', IEEE trans. on PAMI, vol.12, no.2, 131146,1990

[15] P. Whaite, F. Ferrie, 'From uncertainty to visual exploration', IEEE trans. on PAMI, vol. 13, no. 10, 1991.

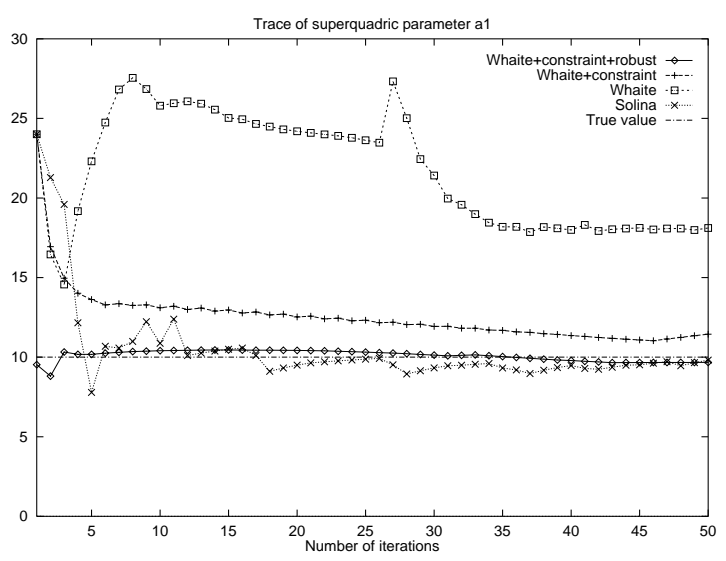

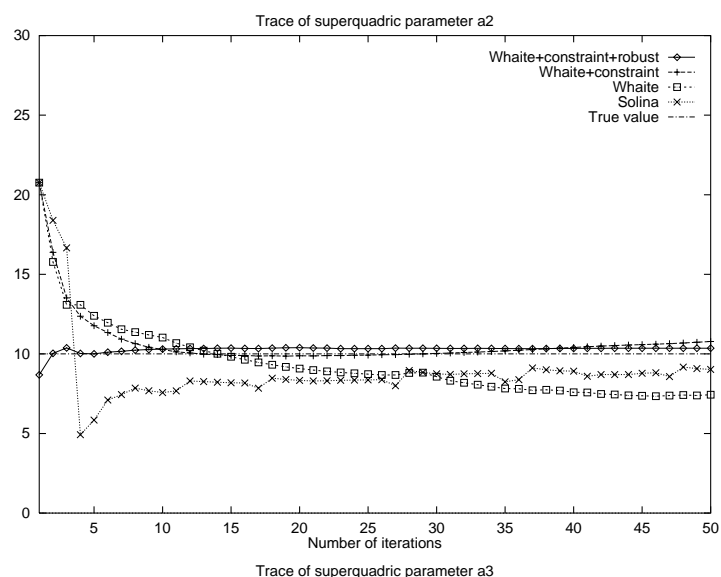
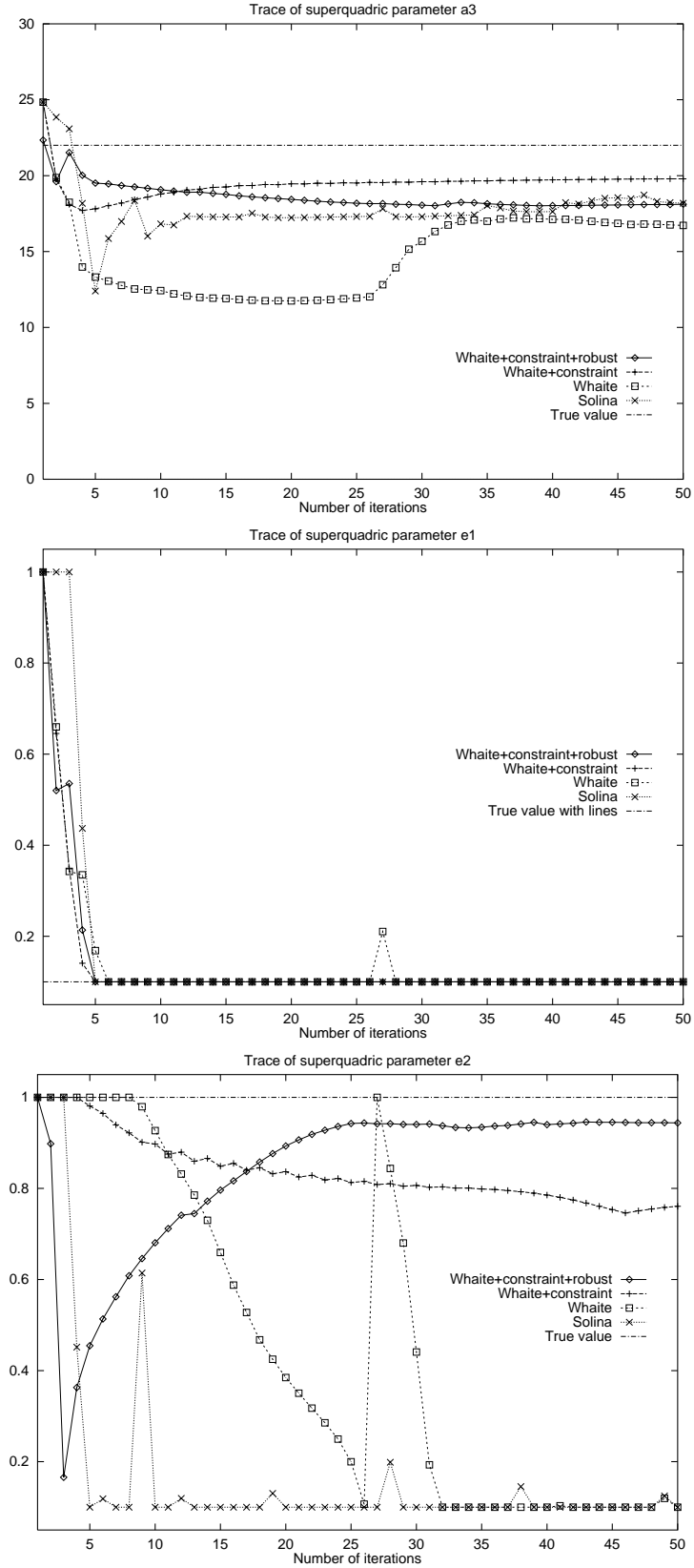

Figure 6: Superquadric parameter traces for various versions of the fitting algorithm applied to elco 2 